\title{
COMBINED CONFOCAL RAMAN MICROSCOPE WITH SCANNING ELECTRON MICROSCOPE; A PARALLEL ANALYSIS OF INORGANIC AND ORGANIC MATERIALS.
}

\author{
Y. Aksenov ${ }^{1}$, A.A. van Apeldoorn ${ }^{1,3}$, J.D. de Bruijn ${ }^{3}$, C.A. van Blitterswijk ${ }^{1,3}$, J.Greve ${ }^{1}$, C.Otto ${ }^{1}$ \\ ${ }^{1}$ University of Twente, Fac. Applied physics, BFT , ${ }^{2}$ University of Twente, Fac. Chemical \\ technology 7500 AE, p.o. box 217, Enschede The Netherlands, ${ }^{3}$ Isotis NV, P.O.box 98, 32723 AB \\ Bilthoven, The Netherlands.
}

Tissue-engineering (TE) has been a fast growing field of research in the past 15 years in industry and science. A major field of interest is the study of seeding osteoprogenitor cells on different biomaterials and subsequently the formation of extra cellular matrix on these materials by cells. We have developed a novel non-invasive technique, which combines a scanning electron microscope (SEM) and a confocal Raman spectroscope CRM in one. High resolution information about sample morphology and it atomic composition are available in modern systems form BSE(back scattered electron) or SE (secondary electron) detectors and from XRMA accordingly. Confocal Raman micro-spectroscopy additionaly to that can provides extremely imporatant information about molecular composition of sample. During the history of development of techniques for micro-analyses a several numbers of ideas were generated and SEM-Raman combination not an exclusion. We will presents here a working integrated scheme of combined CRM-SEM set up and number of application for bioreserach. The CRM-SEM operates as follows; the beam from a diode laser $(\lambda=685 \mathrm{~nm})$ is reflected by a dichroic beam splitter (BS) into the vacuum chamber of a Philips 525 SEM through a coupling window. The beam is then focused by a $100 \mathrm{X}$ microscope objective (Zeiss, West-Germany) parallel to the electron gun on a sample of interest. At first sample is observed by BSE or SE then point of interest is fixied and moved toward adjacent laser focus. The excited Raman scattering is collected by the same microscope objective, passes through the BS and a notch filter, on a confocal pinhole of the system. The scattering is decomposed by a spectrograph system and focused onto a CCD connected with a computer for data collection and analysis by using WinSpec ${ }^{\mathrm{TM}}$ and Microcal $^{\mathrm{TM}}$ Origin $^{\odot}$ data analysis software The system allows for a practical spatial resolution of $\sim 600 \mathrm{~nm}$ with a effective laser power on sample of $\sim 15 \mathrm{~mW}$. Sensitivity of CRM is such that it can measure molecular components of single cell and out-vacuum condition work with a living cell [1]. Results obtained from several research topics indicates a powerful capacities of the tool and are presented. Several topic has been covered in preliminary working phase of given equipment. Characterization of a degradation of bio-degradable materials. Comparison this degradation in-vivo with simulated degradation. Studying products of their interaction with tissue and cells[2]. Facilitation and stimulation of a grow rate for cells and tissue formation for different kind of prosthetic scaffolds made from bio-degradable materials. Therefore, combined capacities of the CRM-SEM provides the possibility to do parallel analysis of inorganic and organic sample constituents. Particularly, an analysis of extra cellular matrix formed by RBMC's using the CRM-SEM shows CaPh and Collagen type I presence, which is not possible to determine by XRMA alone. The presented figures are illustrates that different information including now a molecular analyses available in combined SRM-SEM and different complicated bio-engineered devices can be evaluated in-situ. Obviously that system can work also for such non-biological samples as integrated circuits etc. 
References:

1. Puppels G.J., De Mul, F.F.M., Otto C., Greve J., Robert-Nicoud, M., Arndt-Jovin, D.J., Jovin, T.M. (1990), Nature 347, 301-303.

2. S.A.M. Ali, P.J. Doherty and D.F.Williams, "Molecular biointeractions of biomedical polymerswith extracellular exudate and inflammatory cells and their effects on the biocompatability, in vivo." Biomaterials, 15, 779-785 (1994).
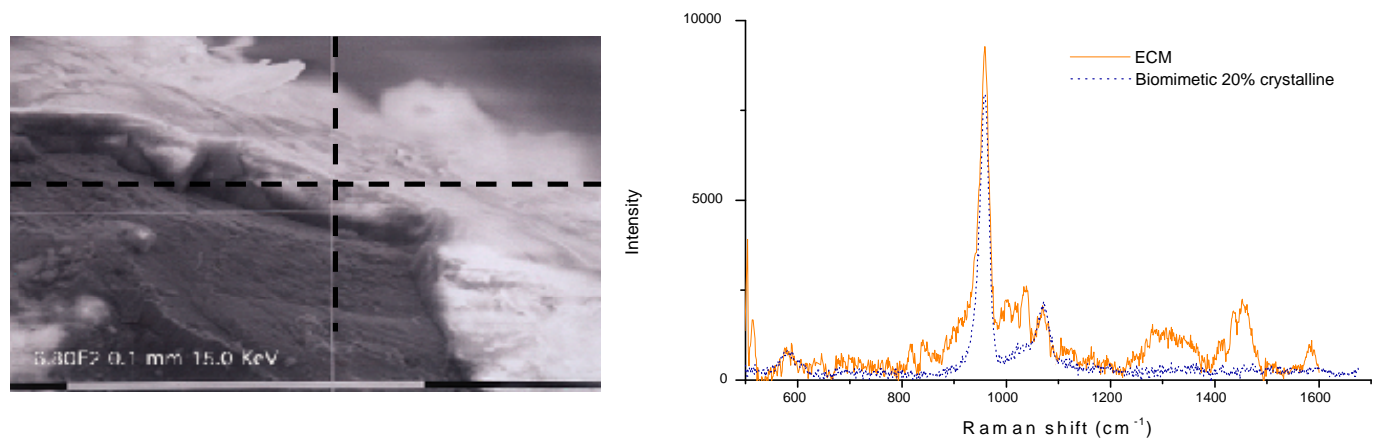

Fig.1 CALB coatings on $\mathrm{Ti}_{6} \mathrm{Al}_{4} \mathrm{~V}$ and it Raman spectrum in cross-mark point. Depends on pike's width a crystallinity of coatings which are facilitating a cell growing or crystalinity of a deposed Calcium Phosphate by cell can be evaluated.
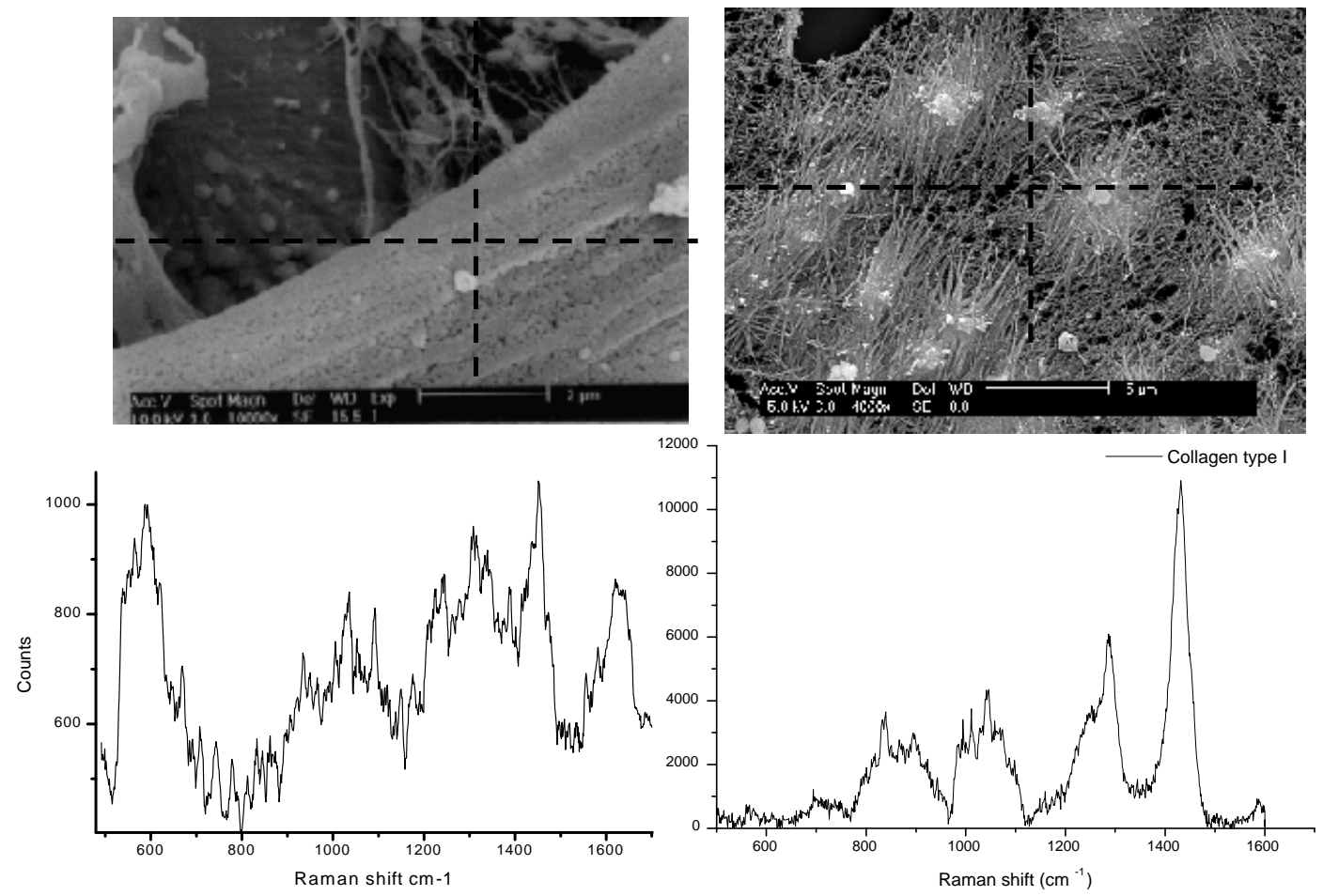

Fig.2. (Left) Image of a bone marrow cell on $\mathrm{Ti}_{6} \mathrm{Al}_{4} \mathrm{~V}$ substrate and it Raman spectrum acquired for $100 \mathrm{sec}$. (Right) Collagen like fibers in extra cellular matrix cultured on normal tissue culture plastic and it Raman spectrum acquired for $200 \mathrm{sec}$.Typical $\mathrm{CH}$-vibration for protein has band in region $1400-1500 \mathrm{~cm}^{-1}$ and it's nicely recognized both in-cellular location and out-cellular matrix. 